\title{
El colgajo romboidal de Limberg en el tratamiento del quiste mucoide
}

\author{
I. Proubasta Renart ${ }^{(1)}$, C. Lamas Gómez ${ }^{(1)}$, J. Itarte Pujals $^{(1)}$, \\ O. Buezo Rivero ${ }^{(2)}$ \\ Hospital de la Santa Cruz y San Pablo. Barcelona. Servicio de Cirugía Ortopédica \\ Y TRAUMATOLOGÍA \\ (1) Consultor Unidad de Cirugía de la Mano y Extremidad Superior \\ (2) MÉDICO RESIDENTE DE TERCER AÑO
}

\author{
Correspondencia: \\ Dr. Ignacio Proubasta Renart. \\ Hospital de la Santa Cruz y San Pablo \\ Servicio de Cirugía Ortopédica y Traumatología \\ C/ Mas Casanovas, 90 \\ 08025 Barcelona \\ email: iproubasta@santpau.cat
}

Objetivo: Valorar los resultados obtenidos mediante el colgajo romboidal de Limberg en pacientes con quiste mucoide.

Material y Método: Se analizan un total de 30 pacientes (18 mujeres, 12 hombres) con quiste mucoide situado en el dorso de la articulación interfalángica distal. En todos los casos el tratamiento fue el mismo, mediante la resección en bloque del quiste y piel suprayacente, y cubrimiento del defecto mediante colgajo romboidal de Limberg. El seguimiento mínimo de estos pacientes fue de 13 meses, con un mínimo de 6 meses y un máximo de 32 meses.

Resultados: Todos los pacientes estuvieron satisfechos con el resultado cosmético conseguido. No hubo que lamentar ningún caso de recidiva, infección ni de necrosis cutánea.

Conclusiones: Dentro de las opciones quirúrgicas existentes para el tratamiento del quiste mucoide, creemos que el colgajo romboidal de Limberg, por su sencillez, resolución y aspecto estético, constituye una alternativa válida en este tipo de patologías.

Palabras clave: quiste mucoide, interfalángica distal, colgajo de piel.
Purpose: To value the results obtained by means Limberg rhomboid flap in patients with mucous cyst.

Material and method: A total of 30 patients (18 women, 12 men) with mucous cyst in the dorsum of the distal interphalangeal joint were analized. In all cases the treatment was the same, by means resection in block of the cyst and supralying skin, and cap of the defect by means Limberg skin flap. The mean follow-up was 13 months, with a minimum of 6 months and a maximum of 32 months. Results: All the patients were satisfied with the cosmetic result. There was no recurrence, infection nor necrosis.

Conclusions: Within the existing surgical options for the treatment of the cyst mucoide, we think that colgajo rhomboid of Limberg, by its simplicity, resolution and aesthetic aspect, constitutes a valid alternative in this type of pathologies.

Key words: mucous cyst, distal interphalangeal joint, skin flap.

Rev. Iberam. Cir. Mano - Vol. 36 • Núm. 2 Noviembre 2008 (107-112) 


\section{INTRODUCCIÓN}

L os gangliones situados a nivel del dorso de las articulaciones interfalángicas distales de los dedos de la mano reciben el nombre de quistes mucoides. Aunque aún no se sabe con certeza la verdadera etiología de los mismos, parece ser que estas lesiones se asocian a menudo con algún grado de $\operatorname{artrosis}^{1-4}$

En cuanto al tratamiento se refiere, existe una gran variedad de técnicas, desde el vaciado del quiste por aspiración mediante aguja ${ }^{5}$, transfixión percutánea con hilos ${ }^{6}$, infiltración con corticoides ${ }^{7}$, aplicación de criocirugía ${ }^{8}$ hasta la exéresis quirúrgica, ya sea ésta realizada percutáneamente mediante tenotomo ${ }^{9} \mathrm{o}$ por cirugía abierta. Sin embargo, esta última modalidad de tratamiento puede realizarse también mediante diversas técnicas, desde la simple exéresis del quiste ${ }^{10}$, exéresis y cierre de la brecha capsular de la interfalángica distal ${ }^{11}$, exéresis y resección de los osteofitos presentes ${ }^{12,13}$, hasta la exéresis y cobertura cutánea mediante colgajos de rotación ${ }^{14}$.

En el presente trabajo se presenta una serie de pacientes con quistes mucoides de los dedos que fueron tratados consecutivamente mediante el colgajo romboidal de Limberg ${ }^{15}$.

\section{MATERIAL Y MÉTODO}

En el periodo comprendido entre enero de 2004 a diciembre de 2007, el colgajo romboidal de Limberg fue empleado en una serie consecutiva de 30 pacientes con quiste mucoide localizado en el dorso de las articulaciones interfalángicas distales de los dedos de la mano. Del total de pacientes, 18 fueron mujeres y 12 hombres. La edad media fue de 46 años para las mujeres (rango: 32-60) y de 64 años para los hombres (rango: 50-82). El dedo índice fue el más frecuentemente afectado (17 casos), seguido del medio (7 casos), anular (4 casos) y pulgar ( 2 casos). En nueve casos $(30 \%)$, se pudo observar la presencia de estriaciones longitudinales de la uña. El estudio radiográfico, mediante proyecciones antero-posterior y lateral del dedo afecto, mostró cambios artrósicos con osteofitos dorsales en 13 casos (43\%).

En cuanto a la técnica quirúrgica, mediante anestesia troncular digital y con isquemia realizada con un dedil de guante quirúrgico cortado en su punta (Figura 1), se diseña el colgajo. En la zona donde asienta el quiste mucoide, se dibuja un rombo equilátero con dos ángulos menores de $60^{\circ}$ y dos mayores de $120^{\circ}$ (Figura 2). El eje del romboide debe diseñarse de acuerdo a dos factores. Si el quiste es oblongo,

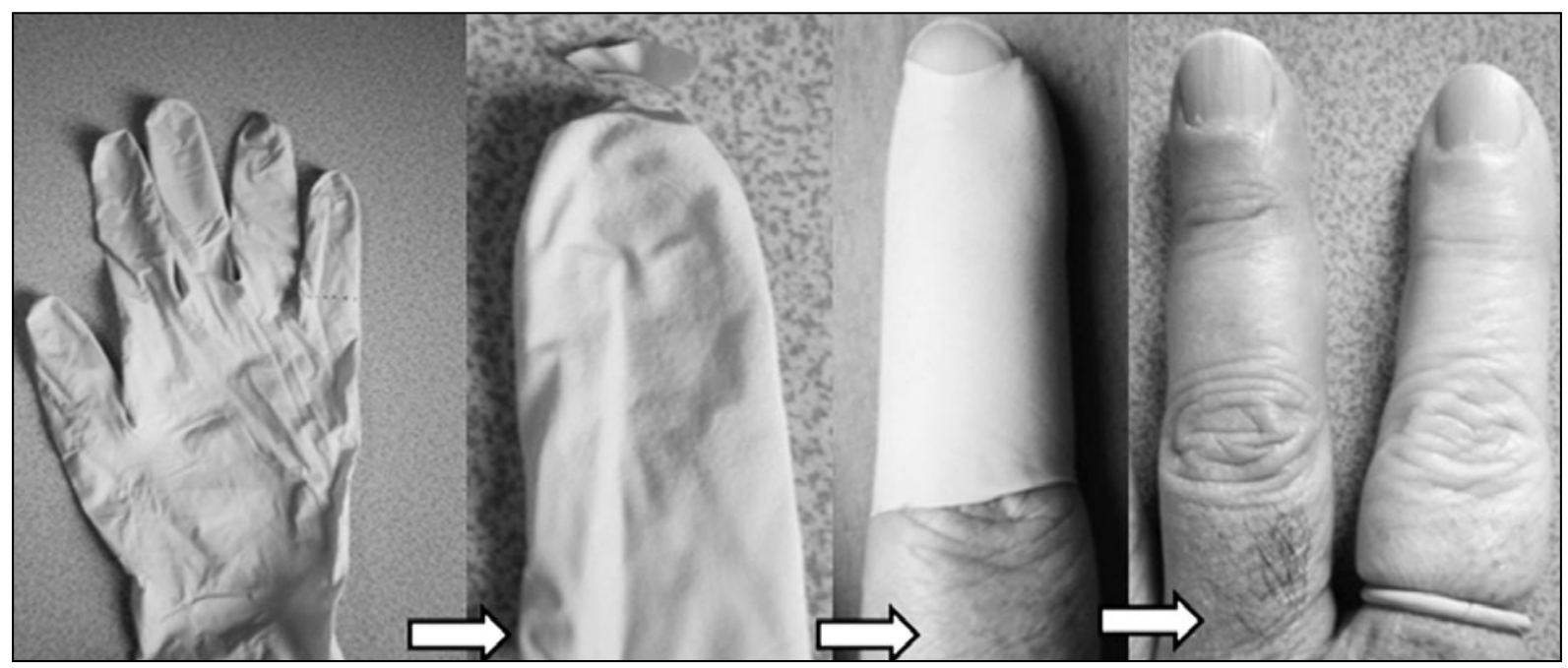

Figura 1: Isquemia del dedo mediante un dedil de guante quirúrgico cortado en su punta. 


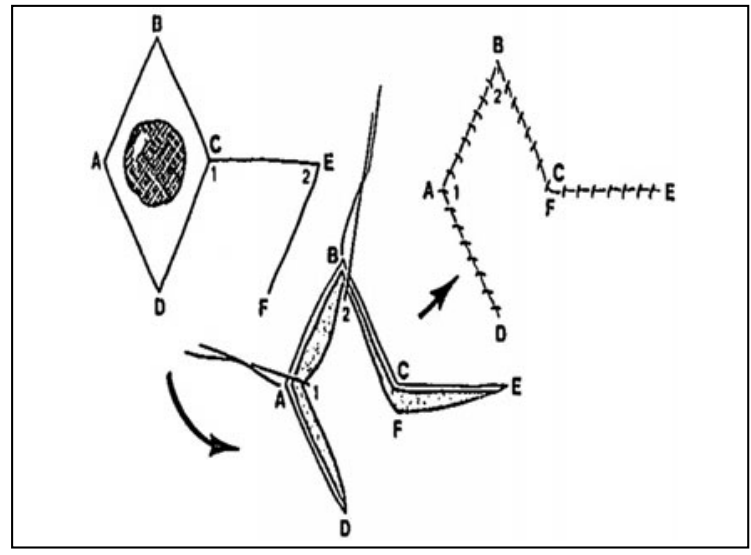

Figura 2: Diseño del colgajo romboidal de Limberg ${ }^{15}$. Todos los lados, tanto del rombo como del colgajo deben tener la misma longitud. El colgajo se diseña a partir de la prolongación de la diagonal A-C construyendo así la línea C-E de la misma longitud que los lados. Luego se traza una línea paralela a C-D determinando así la segunda línea del colgajo E-F también de la misma longitud. La línea de movimiento del tejido va de F hacia C. Una vez que el colgajo ha rotado, el punto de máxima tensión queda localizado entonces en el lugar de cierre del colgajo.

la diagonal más corta del romboide debe asentar a lo largo de la anchura del quiste, mientras que si el mismo es redondo, dicha diagonal debe descansar a lo largo de la máxima extensibilidad de la piel ${ }^{16}$. En cuanto a su ejecución, el área del colgajo se construye trazando una prolongación de la diagonal menor del rombo hacia el área donante, la cual no debe tener una mayor longitud que dicha diagonal, y del extremo de ésta se traza un recta paralela al lado del rombo de la misma longitud que esta última. Por lo tanto, el colgajo está diseñado para cubrir un defecto del mismo tamaño y forma que el quiste. A continuación se realiza la exéresis en bloque del quiste, incluyendo la piel suprayacente $\mathrm{y}$, seguidamente, se diseca el área del colgajo hasta su base para finalmente hacerlo rotar hacia la zona donde previamente estaba el quiste (Figura 2). En ningún caso con artrosis de la interfalángica distal se actuó sobre la articulación ni se resecaron los osteofitos marginales. Seguidamente, se realizan las suturas de piel con prolene 5/0. Una vez completadas las mismas, se retira la isquemia y se observa la viabilidad del colgajo. En caso de existir alguna zona blanquecina indicativa de isquemia, se retira el punto o los puntos corres- pondientes. En cuatro casos de nuestra serie, se tuvo que retirar alguno de los puntos al existir déficit de irrigación del colgajo. Finalmente se coloca una pequeña gasa vaselinada (Linitul $®$ ) sobre la herida quirúrgica y se la cubre con una venda confeccionada con una gasa y, encima de la misma, un vendaje de Coban ${ }^{\circledR}$ para evitar el edema del dedo. A los 4-5 días se revisa la herida, colocándose un apósito simple de gasa envuelta también con venda de Coban. A los 12 días se retiran los puntos, permitiendo al paciente todo tipo de actividad, incluyendo la higiene de la mano. En ningún caso recomendamos la povidona yodada o cualquier antiséptico sobre la herida, por cuanto interesa que la piel superficial se descame lo más rápidamente posible para permitir una rápida regeneración de la epidermis subyacente.

\section{RESULTADOS}

El seguimiento medio de estos pacientes fue de 13 meses, con un mínimo de 6 meses y un máximo de 32 meses. En ningún caso se reprodujo la lesión. Asimismo, todos los pacientes estuvieron satisfechos con el resultado cosmético conseguido. No hubo que lamentar ningún caso de infección ni de necrosis cutánea (Figura 3).

\section{DISCUSIÓN}

El quiste mucoide de los dedos de la mano constituye una patología relativamente frecuente y se le asocia a menudo con la artrosis de la articulación interfalángica distal ${ }^{17}$, aunque se observa también después de un traumatismo, especialmente en pacientes de menos de 40 años $^{18}$. Tiene una predilección sobre el sexo femenino, y su presentación suele ser más común entre los 50-70 años de edad, tal como así ocurrió en nuestros casos. En general, se localizan siempre en el dorso de las articulaciones interfalángicas distales, subcutáneos y recubiertos por una piel muy fina. A pesar de que la mayoría de ocasiones los quistes son de pequeño tamaño, desde unos milímetros a un centímetro de diámetro, suelen oca- 


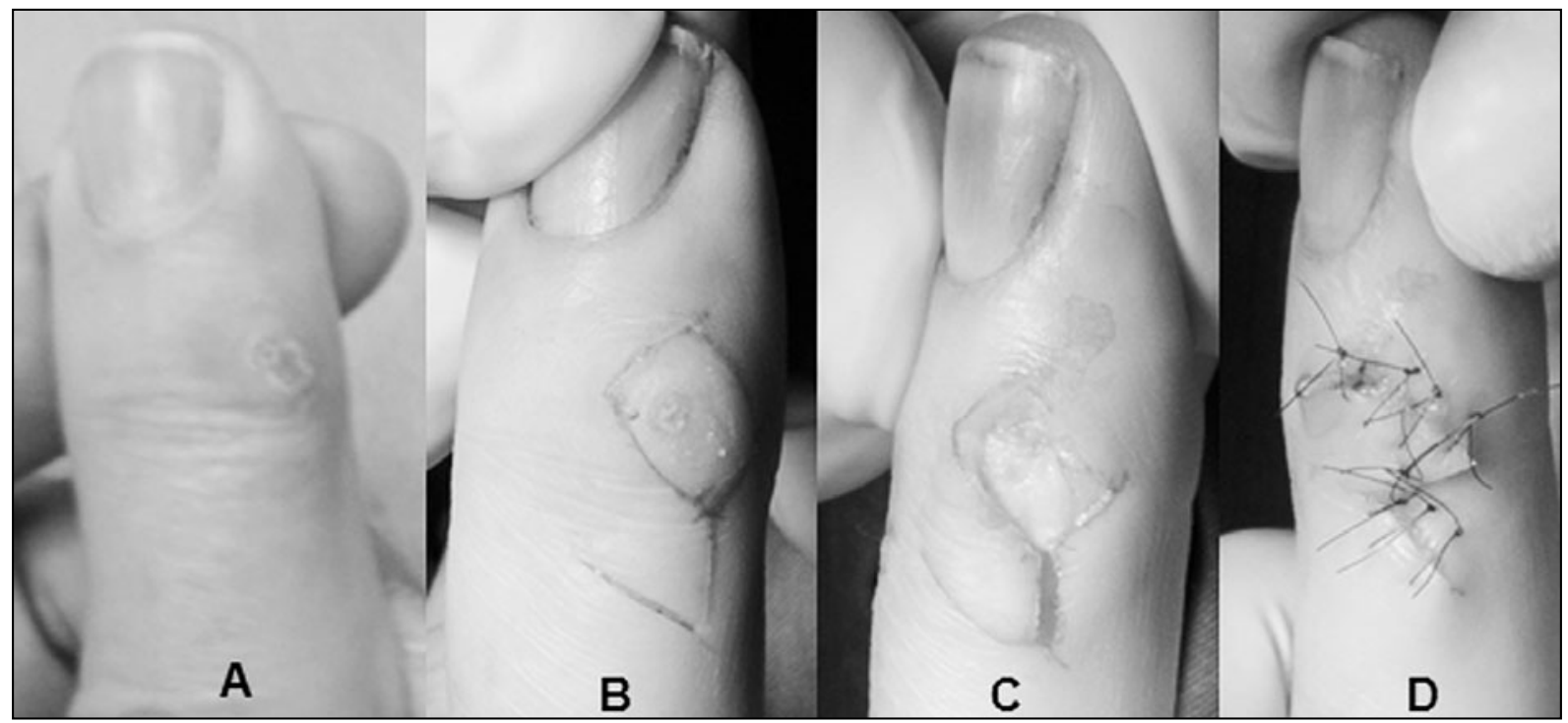

Figura 3: Resultado obtenido después de proceder al colgajo de Limberg ${ }^{15}$ en un paciente portador de un quiste mucoide. A) Aspecto inicial. B) Diseño del colgajo. C) exéresis del quiste y de la piel suprayacente. D) Aspecto final.

sionar muchas molestias ${ }^{7,10,19}$. Asimismo es común observar, incluso antes de que aparezca el quiste, la aparición de estrías longitudinales de la uña, lo que indica una afectación más o menos importante de la matriz üngueal. Esta estriación üngueal aparece en un tercio de los $\operatorname{casos}^{10}$, cifra similar a la observada en nuestra serie. En ocasiones puede ulcerarse, vaciando su contenido al exterior. Tampoco es infrecuente su infección. En las preparaciones histológicas se observa que la epidermis y dermis de la piel que recubre el quiste, está infiltrada por numerosos conductos y/o lagunas satélites, rellenos de material mucinoso. Es por ello, que numerosos cirujanos, incluidos nosotros, indican la exéresis del quiste y toda la piel que lo recubre para evitar su recidiva ${ }^{20}$.

Debido a que en un número considerable de casos el quiste se asocia con la presencia de cambios artrósicos con la formación de osteofitos dorsales que protruyen hacia la piel, es común hacer una distinción entre los quistes con cambios degenerativos de la articulación de aquellos que no los presentan ${ }^{21}$.

En cuanto a la evolución natural del quiste, una vez aparece, raramente se resuelve espontáneamente. Sin embargo, en un estudio longitudinal se sugiere que alguno de ellos desaparece gradualmente con el tiempo ${ }^{7}$.
Por lo que se refiere al tratamiento, existen varias modalidades, unas conservadoras y otras quirúrgicas. Sin embargo, los tratamientos conservadores tienen un gran número de recidivas. En este sentido, Nelson et al. ${ }^{3}$ reportaron un $34 \%$ de recidivas sobre 44 pacientes tratados mediante vaciado con aguja y expresión del contenido quístico, y un 60\% sobre 145 pacientes tratados mediante aspiración e infiltración con cortidoides. En cuanto al tratamiento quirúrgico, la mayoría de autores combinan la exéresis del quiste con el desbridamiento de la articulación, incluyendo en la misma la resección de los osteofitos si éstos están presentes ${ }^{2,4,12}$, hecho que puede representar hasta el $78 \%$ de los casos, aún cuando en nuestra serie fue de solo el 43\%. Con esta técnica el índice de recidivas es mínimo. Así Eaton et al. ${ }^{2}$, sobre un total de 44 quistes, sólo tuvieron un caso de recidiva, y Kasdan et al. ${ }^{12}$, sobre 178 pacientes, reportaron dos casos de recidivas y otros dos casos que se complicaron con una infección. A pesar de estos buenos resultados, la recuperación de la movilidad de las interfalángicas distales, especialmente la flexión, es a menudo muy prolongada y no siempre completa ${ }^{18}$. Es por ello que algunos autores, incluidos nosotros, no consideran imprescindible el desbridamiento articular y más cuando no existen signos radiográfi- 


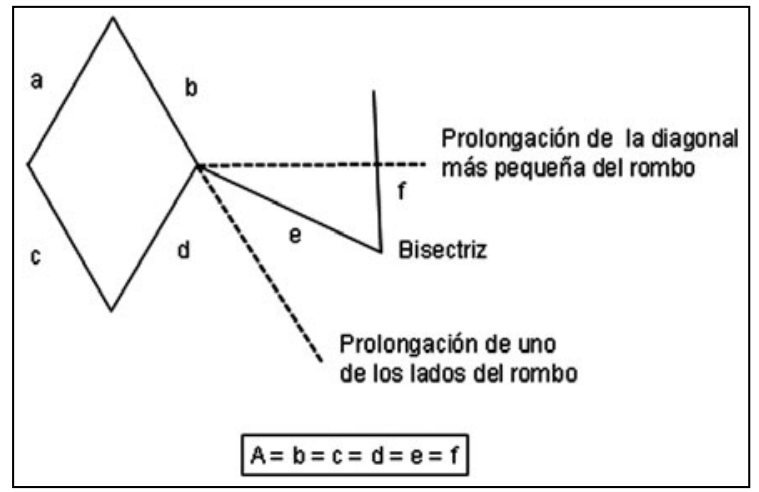

Figura 4. Colgajo de Dufourmentel ${ }^{28}$ (ver texto).

cos de artrosis de la interfalángica distal. De hecho, se ha podido demostrar que no es necesaria la resección de los osteofitos para conseguir un buen resultado ${ }^{22}$.

Lo que sí coinciden todos los autores y también nosotros, es en la necesidad de resecar toda la piel que recubre el quiste, pues la simple exéresis del mismo sin la correspondiente resección de la piel, produce un alto índice de recidivas $^{2}$. Al respecto, si bien puede procederse a la sutura primaria después de la resección conjunta de quiste y piel cuando el mismo es de pequeño tamaño, en la mayoría de casos ello no es posible, haciéndose necesario la colocación de un injerto libre de piel ${ }^{18}$ o un colgajo rotacional $^{14}$. Es en esta última modalidad de tratamiento donde existen un sinfín de colgajos rotacionales, aunque todos ellos derivados del preconizado por Kleinert et al. ${ }^{2}$ en 1972. Sin embargo, la mayoría de ellos van acompañados de un desbridamiento articular con resección de los osteofitos, lo que puede dar lugar, tal como se ha comentado, a una lesión de la matriz üngueal y/o a una pérdida de la movilidad de la articulación interfalángica distal, especialmente la flexión.

El colgajo romboidal de Limberg, que es el que hemos utilizado en nuestros pacientes, fue descrito por primera vez por el cirujano ruso Alexander Limberg en 1946 y publicado en inglés, en $1964^{15}$. Si bien dicho colgajo fue empleado inicialmente para cubrir los defectos cutáneos después de la exéresis de neoplasias cutáneas, especialmente de la $\operatorname{cara}^{23}$, en la actualidad se utiliza en multitud de regiones del cuerpo con las mismas indicaciones de cobertura. Así, se ha utilizado en los recubrimientos de piel tras mastectomías parciales ${ }^{24}$, quistes pi- lonidales ${ }^{25} \mathrm{y} / \mathrm{o}$ úlceras sacras por decúbito ${ }^{26}$. A nivel de la mano, especialmente después de la resección de quistes de los dedos, se encuentra el trabajo de Imran et al. ${ }^{27}$ quienes lo emplearon en 9 pacientes, seis de ellos con quiste mucoide, dos con tumor de células gigantes de vaina tendinosa y uno con histiocitoma. Sus resultados fueron homogéneamente buenos y sin tener ningún caso de recidiva.

En nuestra serie de 30 casos, se siguió el mismo procedimiento que el descrito por Imran et al. ${ }^{27}$, obteniendo también unos excelentes resultados. Sin embargo, hemos de comentar algunas pequeñas dificultades, todas ellas derivadas de la tensión del colgajo, lo que obligó en 4 casos a retirar algunos puntos de sutura para garantizar la viabilidad del mismo. Es posible que en alguno de estos casos, la variante del colgajo de Limberg diseñado por Dufourmentel ${ }^{28}$ y bautizado como «colgajo LLL» sea más adecuado, pues permite una mayor versatilidad además de que la tensión de reorientación es menor que el obtenido con el rombo estándar de Limberg. El diseño del colgajo de Dufourmentel consiste en trazar una bisectriz del ángulo formado por la prolongación de uno de los lados con la diagonal más pequeña del rombo. Esta bisectriz, de la misma longitud que los lados del rombo, se continúa con una perpendicular a la diagonal mayor del rombo, que medirá también la misma longitud que la anterior. (Figura 4)

Finalmente, comentar la posibilidad de complicaciones tras la exéresis del quiste, entre las que cabe citar la ya comentada pérdida de movilidad de la articulación inrterfalángica distal, la infección, la lesión de la matriz üngueal con deformidad de la uña y la infrecuente desviación radial y/o cubital de la articulación por lesión de uno de los ligamentos colaterales ${ }^{1,29}$. La recidiva del quiste se sitúa en el $3 \%$ de los $\operatorname{casos}^{18}$.

\section{CONCLUSIONES}

Dentro de las opciones quirúrgicas existentes para el tratamiento del quiste mucoide, creemos que el colgajo romboidal de Limberg, por su sencillez, resolución y aspecto estético, constituye una alternativa válida en este tipo de patologías. 


\section{BIBLIOGRAFÍA}

1. Constant E, Royer JR, Pollard RJ. Mucous cysts of the fingers. Plast Reconstr Surg, 1969; 43: 241-6.

2. Kleinert HE, Kutz JE, Fishman $\mathrm{JH}$, et al. Etiology and treatment of the so-called mucous cyst of the finger. $J$ Bone Joint Surg Am, 1972; 54: 1455-8.

3. Nelson CL, Sawmiller S, Phalen GS. Ganglions of the wrist and hand. J Bone Joint Surg Am, 1972; 54: 1459-64.

4. Eaton RG, Dobranski Al, Littler JW. Marginal osteophyte excision in treatment of mucous cysts. J Bone Joint Surg Am, 1973; 55: 570-4.

5. Epstein E. A simple technique for managing digital mucous cysts. Arch Dermatol, 1979; 115: 1315-6.

6. Dao L. A new method of treatment of ganglions and synovial cysts. J Occup Med, 1964; 6: 217-20.

7. Sonnex TS. Digital myxoid cysts: a review. Cutis, 1986; 37: 89-94.

8. Bardach HG. Managing digital mucoid cysts by cryosurgery with liquid nitrogen: preliminary report. J Dermatol Surg Oncol, 1983; 9: 455-8.

9. Soren A. Pathogenesis and treatment of ganglion. Clin Orthop, 1966; 48: 173-9.

10. Dodge LD, Brown RL, Niebauer JJ. The treatment of mucous cysts: long-term follow-up in sixty-two cases. J Hand Surg Am, 1984; 9: 901-4.

11. de Berker, David MRCP; Lawrence, et al. Ganglion of the distal interphalangeal Joint (Myxoid Cyst): Therapy by
Identification and repair of the leak of joint fluid. Archiv Dermatol, 2001; 137: 607-10.

12. Kasdan ML, Stallings SP, Leis VM. Outcome of surgically treated mucous cysts of the hand. J Hand Surg Am, 1994; 19: 504-7.

13. Gingrass MK, Brown RE, Zook EG. Treatment of fingernail deformities secondary to ganglions of the distal interphalangeal joint. J Hand Surg Am, 1995; 20: 502-5.

14. Shin EK, Jupiter JB. Flap advancement coverage after excision of large mucous cysts. Current Orthop Pract, 2008; 19: 276-9.

15. Limberg AA. Modern trends in plastic surgery: design of local flaps. Mod Trends Plast Surg, 1966; 2: 38-61.

16. Stark HL. Directional variations in the extensibility of human skin. Br J Plast Surg, 1977; 30: 105-14.

17. Loder RT, Robinson JH, Jackson WT, et al. A surface ultrastructure study of ganglia and digital mucous cysts. J Hand Surg Am, 1988; 13: 758-62.

18. Fritz GR, Stern PJ, Dickey M. Complications following mucous cyst excision. J Hand Surg Br, 1997; 22: 222-5.

19. King ESJ. Mucous cysts of the fingers. Aust N Z J Surg, 1995; 21: 121-9.

20. Chen WS, Lin CC. Mucous cyst of the distal interphalangeal joint: treatment by simple excision or excision and rotating flap. J Hand Surg Br, 1991; 16 : 118-9.

21. Zuber TJ. Office management of digital mucous cysts. Am Fam Phys, 2001; 64: 1987-90.

22. Clifford L. Skin excision and osteophyte removal is not required in the surgical treatment of digital myxoid cysts. Arch Dermatol, 2005; 141:1560-4.

23. Borges AF. Chossing the correct Limberg flap. Plast Reconstr Surg, 1971; 62: 542-5.

24. Gwynn BR, Williams CR. Use of the Limberg flap to close breast wounds after partial mastectomy. Ann Royal Coll Surg Engl, 1985; 67: 245-6.

25. Akca T, Colak T, Ustunsoy B, et al. Randomized clinical trial comparing primary closure with the Limberg flap in the treatment of primary sacrococcygeal pilonidal disease. $\mathrm{Br} J$ Surg, 2005; 92:1081-4.

26. Luscher NJ, Kuhn W, Zach GA. Rhomboid flaps in surgery for decubital ulcers: indications and results. Ann Plast Surg, 1986; 16: 415-22.

27. Imran D, Koukkou C, Bainbridge LC. The rhomboid flap: a simple technique to cover the skin defect produced by excision of a mucous cyst of a digit. J Bone Joint Surg Br, 2003; 85: 860-2.

28. Dufourmentel C. Le fermature des partes de substance cutanées limitées, «Le lambeau de rotation en $L$ pour losange, dit LLL». Ann Chir Plast, 1962; 7: 61-6.

29. Brown RE, Zook EG, Russell $R C$, et al. Fingernail deformities secondary to ganglions of the distal interphalangeal joint (mucous cysts). Plast Reconstr Surg, 1991; 87: 718-25. 